\title{
Identification, Mapping and Relative Quantitation of SARS-CoV-2 Spike Glycopeptides by Mass- Retention Time Fingerprinting
}

Chalk, R. ${ }^{1}$, Greenland, W. ${ }^{2}$, Moreira, ${ }^{1} .{ }^{1}$, Coker, J. ${ }^{1}$, Mukhopadhyay S.M.M. ${ }^{1}$, Williams, E. ${ }^{1}$, Manning, $\mathrm{C}^{1}{ }^{1}$, Bohstedt, T. $^{1}$, McCrorie, R. ${ }^{1}$, Fernandez-Cid, A. ${ }^{1}$, Burgess-Brown, N.A. ${ }^{1}$

${ }^{1}$ Centre for Medicines Discovery, ORCRB, Oxford University, OX3 7DQ, UK

${ }^{2}$ Agilent Technologies, Lakeside, Cheadle Royal Business Park, Cheadle, Cheshire, SK8 3GR, UK

\begin{abstract}
We describe a novel analytical method for rapid and robust identification, mapping and relative quantitation of glycopeptides from SARS-CoV-2 Spike protein. The method may be executed using any LC-TOF mass spectrometer, requires no specialised knowledge of glycan analysis and makes use of the differential resolving power of reversed phase HPLC. While this separation technique resolves peptides with high efficiency, glycans are resolved poorly, if at all. Consequently, glycopeptides consisting of the same peptide bearing different glycan structures will all possess very similar retention times and co-elute. While this has previously been viewed as a disadvantage, we show that shared retention time can be used to map multiple glycan species to the same peptide and location. In combination with MSMS and pseudo MS3, we have constructed a detailed mass-retention time database for Spike. This database allows any ESI-TOF equipped lab to reliably identify and quantify spike glycans from a single overnight elastase protein digest in less than 90 minutes.
\end{abstract}

Key words:

SARS-CoV-2, Spike, RBD, Glycoprotein, Glycopeptide, Glycan, Mass Spectrometry, HPLC, Database

\section{Introduction}

Glycosylation is known to play an important role in the efficacy and antigenicity of therapeutic proteins [1-3]. The current SARS-CoV-2 pandemic has spurred urgent research, much of it devoted to preparing vaccines, therapeutic antibodies or antibody tests based on Spike protein, the virus's primary surface antigen [4]. This $145 \mathrm{kDa}$ protein forms a trimer [5] with each subunit bearing twenty-two potential $\mathrm{N}$-linked glycosylation sites and two O-linked sites of which approximately seventeen are occupied [5]. The unusually heavy and complex glycosylation observed in Spike protein is believed to play an important role in the pathogenicity of SARS-CoV-2 by mimicking host cell glycans and allowing the virus to evade the normal immune response [6]. Analysis of expressed Spike protein by mass spectrometry presents unique challenges in terms of its size and the number and complexity of its glycans. These challenges have been commendably met to date by laboratories with wide experience in glycan analysis and access to very sensitive, high-end nano-LC-MSMS mass spectrometers [1, 7-9]. However, in our laboratory and in others a rapid and more robust methodology is needed for routine analysis of different batches of expressed Spike protein. In addition, any method which is reliant on LC-MSMS of glycopeptides may not necessarily detect specific glycans which fail to fragment under the conditions selected. LC-MS, by contrast, generates a mass, retention time and relative abundance for all ionizable species. We have developed a simple Mass-Retention Time Fingerprinting (MRTF) method for rapid and robust identification, mapping and relative quantitation of Spike glycans. Overnight digestion using a single enzyme followed by a 65minute LC-MS run using any accurate mass instrument are the only experimental requirements. The resulting LC-MS data contains accurate mass, retention time and relative abundance values for each glycopeptide component. This dataset needs only to be matched against the pre-existing Spike glycopeptide database reported here, as shown in Figure 1. We describe this method as "analytical mode", which is both conceptually simple to understand, and straightforward to implement in a typical mass spectrometry laboratory. For scientific completeness, we also describe the "discovery mode" which we have used to generate the data for our Mass-Retention Time Fingerprinting 
database. We stress, however, that there is no requirement for users to duplicate this discovery mode. The analytical mode in conjunction with the database we have provided is all that is necessary to characterise Spike glycans by MRTF.

Figure 1. Mass-Retention Time Fingerprint analysis of Spike glycans

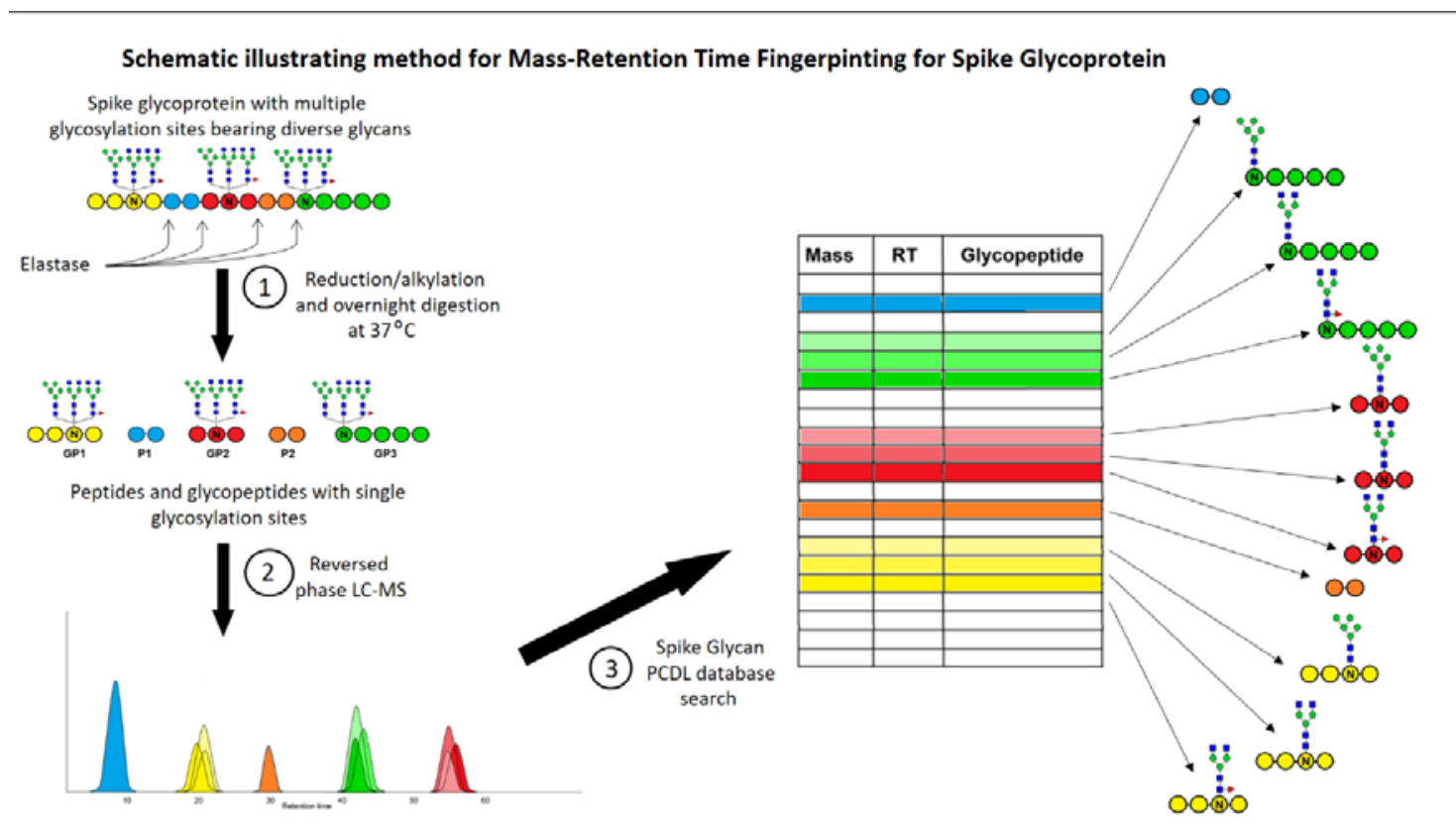

Results

Combined extracted ion chromatograms are illustrated in Figure 2. It can be seen that some species (peptides) are completely resolved, while many other species (glycans) co-elute.

Figure 2. Combined Extracted lon Chromatogram (EIC) for 140 observed Spike glycopeptides separated by reversed phase LC-MS and identified by Mass-Retention Time Fingerprinting (MRTF)

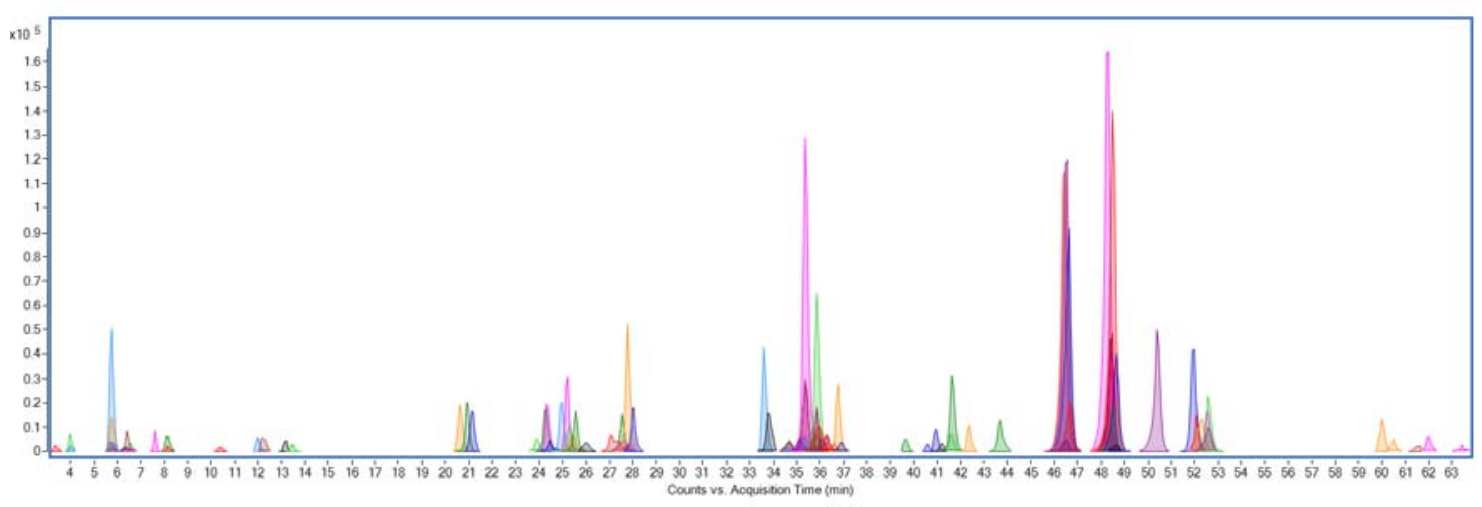

We observed one hundred and forty glycopeptides by LC-MS. These are recorded grouped by ascending retention time in Tables $1 \mathrm{a}$ and $1 \mathrm{~b}$ along with accurate masses, peptide sequences, glycan assignments and a key to the glycan structures. The location of each glycopeptide series on Spike is indicated in the first column. It may be seen that all observed glycans for the same peptide occur within a four minute retention time window. Accurate mass and estimated retention time are included for a further three hundred and six glycopeptides. 
bioRxiv preprint doi: https://doi.org/10.1101/2020.07.24.217562; this version posted July 27, 2020. The copyright holder for this preprint (which was not certified by peer review) is the author/funder, who has granted bioRxiv a license to display the preprint in perpetuity. It is made available under aCC-BY-ND 4.0 International license.

Table 1a. Spike elastase glycopeptide mass retention time database (PCDL) containing data for 140 observed glycopeptides and data for a further 306 inferred glycopeptides (RT 2-32 min)

\begin{tabular}{|c|c|c|c|c|}
\hline $\begin{array}{l}\text { Glycan } \\
\text { posn. }\end{array}$ & RT (min) & Mass & Glycopeptide & $\begin{array}{c}\text { Observed/ } \\
\text { Inferred }\end{array}$ \\
\hline \multirow{11}{*}{ N343 } & 2.4 & 2151.9206 & VFNATRF GO & Inf \\
\hline & 2.4 & 2297.9785 & VFNATRF GOF & Inf \\
\hline & 2.4 & 1421.6562 & VFNATRF Man1 & Inf \\
\hline & 2.4 & 1583.709 & VFNATRF Man2 & Inf \\
\hline & 2.4 & 1907.8146 & VFNATRF Man4 & Int \\
\hline & 2.4 & 2069.8674 & VFNATRF Man5 & Int \\
\hline & 2.4 & 2231.9202 & VFNATRF Man6 6 & Inf \\
\hline & 2.4 & 2393.973 & VFNATRF Man? & Inf \\
\hline & 2.4 & 2556.0258 & VFNATRF Man8 & Inf \\
\hline & 2.4 & 2718.0786 & VFNATRF Man9 & Int \\
\hline & 2.464 & 1745.7618 & VFNATRF Man3 3 & Obs \\
\hline \multirow{11}{*}{ N8O1 } & 3.3 & 1792.6885 & NFSQGO & Int \\
\hline & 3.3 & 1938.7464 & NFSQ GOF & Inf \\
\hline & 3.3 & 1872.6881 & NFsQ Man6 & Inf \\
\hline & 3.3 & 2034.7409 & NFSQ Man7 & Inf \\
\hline & 3.3 & 2196.7937 & NFSQ Man8 & $\operatorname{lnf}$ \\
\hline & 3.3 & 2358.8465 & NFSQ Man9 & Int \\
\hline & 3.364 & 1710.6353 & NFSQ Mans & obs \\
\hline & 3.8 & 1062.4241 & NFSQ Man1 & Inf \\
\hline & 3.8 & 1224.4769 & NFSQ Man2 & Inf \\
\hline & 3.888 & 1548.5825 & NFSQ Man 4 & obs \\
\hline & 3.898 & 1386.5297 & NFSQ Man3 & Obs \\
\hline \multirow{14}{*}{ 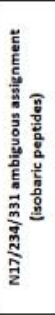 } & 5.636 & 2210.8192 & NL/IT Man9 & Obs \\
\hline & 5.7 & 752.344 & NL//T (GleNAC)2 & $\operatorname{lnf}$ \\
\hline & 5.7 & 549.2646 & NL/IT GICNAC stump & Inf \\
\hline & 5.7 & 1644.6612 & NL/IT GO & Inf \\
\hline & 5.7 & 1790.7191 & NL/IT GOF & Inf \\
\hline & 5.7 & 914.3968 & NL/IT Man1 & $\operatorname{lnf}$ \\
\hline & 5.7 & 1076.4496 & NL//T Man2 & Int \\
\hline & 5.7 & 1238.5024 & NL/IT Man3 & $\operatorname{lnf}$ \\
\hline & 5.7 & 346.1852 & NU/IT peptide & Inf \\
\hline & 5.737 & 2048.7664 & NL/IT Man8 & Obs \\
\hline & 5.751 & 1886.7136 & NL/TT Man7 & obs \\
\hline & 5.761 & 1724.6608 & NL/IT Man6 & Obs \\
\hline & 5.821 & 1562.608 & NU//T Man5 & obs \\
\hline & 6.549 & 1400.5552 & NL/IT Man4 & obs \\
\hline \multirow{11}{*}{ N8O1 } & 6.4 & 2187.8412 & DFGGFNFS GO & Inf \\
\hline & 6.4 & 2333.8991 & DFGGFNFS GOF & Inf \\
\hline & 6.4 & 1457.5722 & DFGGFNFS Man1 & Inf \\
\hline & 6.4 & 1619.625 & DFGGFNFS Man2 & Inf \\
\hline & 6.4 & 1781.6778 & DFGGFNFS Man3 & Inf \\
\hline & 6.4 & 1943.7352 & DFGGFNFS Man4 & Int \\
\hline & 6.4 & 2267.8408 & DFGGFNFS Man6 & Inf \\
\hline & 6.4 & 2429.889 & DFGGFNFS Man7 & Inf \\
\hline & 6.4 & 2591.9418 & DFGGFNFS ManB & Inf \\
\hline & 6.4 & 2753.9946 & DFGGFNFS Man9 & Inf \\
\hline & 6.454 & 2105.7834 & DFGGFNFS ManS & Obs \\
\hline & 6.537 & 1725.6561 & NKS Man6 & Obs \\
\hline & 6.554 & 1563.6033 & NKS Man5 & obs \\
\hline & 7.2 & 1645.6565 & NKS GO & Int \\
\hline & 7.2 & 1791.7144 & NKS GOF & Inf \\
\hline & 7.2 & 915.3921 & NKS Man1 & Inf \\
\hline N149 & 7.2 & 1077.4449 & NKS Man2 & Inf \\
\hline N149 & 7.2 & 1239.4977 & NKS Man3 & Inf \\
\hline & 7.2 & 1401.5505 & NKS ManA & Inf \\
\hline & 7.2 & 1887.7089 & NKS Man7 & Int \\
\hline & 7.2 & 2049.7617 & NKS Man8 & Inf \\
\hline & 7.2 & 2211.8145 & NKS Man9 & Inf \\
\hline & 7.271 & 1563.6033 & NKS Man5 & Obs \\
\hline & 7 & 2323.9062 & YNENGTITD GO & Inf \\
\hline & 7 & 2469.9641 & YNENGITD GOF & Inf \\
\hline & 7 & 2485.959 & YNENGTITD G1 & Inf \\
\hline & 7 & 2632.0169 & YNENGIITD G1F & Inf \\
\hline & 7 & 2794.0697 & YNENGTITD G2F & Inf \\
\hline & 7 & 1593.6418 & YNENGIITD Man1 & Inf \\
\hline & 7 & 1755.6946 & YNENG GITD Man2 2 & Inf \\
\hline N282 & 7 & 1917.7474 & YNENGTITD Man3 & Int \\
\hline & 7 & 2079.8002 & YNENGTITD Man4 & Inf \\
\hline & 7 & 2241.853 & YNENG GITD ManS & Inf \\
\hline & 7 & 2403.9058 & YNENGTITD Man6 & Inf \\
\hline & 7 & 2565.9586 & YNENGIITD Man? & Inf \\
\hline & 7 & 2728.0114 & YNENGTITD Man8 & Inf \\
\hline & 7 & 2890.0642 & YNENGTITD Man9 & $\operatorname{lnf}$ \\
\hline & 7.054 & 2648.0118 & YNENGTITD G2 & Obs \\
\hline & 8.082 & 2120.8366 & NGTITDAVD Man5 & obs \\
\hline & 8.144 & 2444.9422 & NGIITDAVO Men? & obs \\
\hline & 8.2 & 2202.8898 & NGTITDAVD GO & Inf \\
\hline & 8.2 & 2348.9477 & NGTITDAVD GOF & Inf \\
\hline & 8.2 & 1472.6254 & NGTITDAVD Man1 & Inf \\
\hline N282 & 8.2 & 1634.6782 & NGTITDAVD Man2 & Inf \\
\hline & 8.2 & 1796.731 & NGTITDAVD Man3 & Inf \\
\hline & 8.2 & 1958.7838 & NGTITDAVD Man4 & Inf \\
\hline & 8.2 & 2606.995 & NGIITOAVD Man8 & Inf \\
\hline & 8.2 & 2769.0478 & NGTITOAVD Man9 & Inf \\
\hline & 8.239 & 2282.8894 & NGIITDAVD Man6 & Obs \\
\hline
\end{tabular}

\begin{tabular}{|c|c|c|c|c|}
\hline $\begin{array}{l}\text { Glycan } \\
\text { posn. }\end{array}$ & RT $(\min )$ & Mass & Glycopeptide & $\begin{array}{c}\text { Observed// } \\
\text { Inferred }\end{array}$ \\
\hline & 10.3 & 1876.746 & PINFT GO & $\operatorname{lnf}$ \\
\hline & 10.3 & 2022.8039 & PTNFT GOF & Int \\
\hline & 10.3 & 2038.7988 & PTNFT G1 & Inf \\
\hline & 10.3 & 1146.4816 & PINFT Man1 & Inf \\
\hline & 10.3 & 1308.5344 & PTNFT Man2 & Inf \\
\hline & 10.3 & 1470.5872 & PTNFT Man3 & Inf \\
\hline & 10.3 & 1632.64 & PTNFT Man4 & Inf \\
\hline N717 & 10.3 & 1794.6928 & PTNFT Mans & Inf \\
\hline & 10.3 & 1956.7456 & PINFT Man6 & $\operatorname{lnf}$ \\
\hline & 10.3 & 2118.7984 & PINFT Man7 & Inf \\
\hline & 10.3 & 2280.8512 & PTNFT Man8 & Inf \\
\hline & 10.3 & 2442.904 & PTNFT Mang & Int \\
\hline & 10.365 & 2184.8567 & PINFT G1F & Obs \\
\hline & 10.4 & 2200.8516 & PTNFT G2 & Int \\
\hline & 10.448 & 2346.9095 & PTNFT G2F & Obs \\
\hline & 11.995 & 2533.9915 & CLIGAEHVNNS Man6 & Obs \\
\hline & 12.1 & 2453.9919 & CLIGAEHVNNS GO & Inf \\
\hline N657 & 12.1 & 2600.0498 & CUIGAEHVNNS GOF & Inf \\
\hline & 12.105 & 2371.9387 & CLIGAEHVNNS Man5 & Obs \\
\hline & 12.204 & 2209.8859 & CUGAEHVNNS Man4 & Obs \\
\hline & 13.161 & 2825.0954 & EFRWYSSANNCT Man6 & Obs \\
\hline & 13.4 & $2 / 45.0958$ & EFRUYSSANNCT GO & Inf \\
\hline & 13.4 & 2891.1537 & EFRYYSSANNCT GOF & Inf \\
\hline & 13.4 & 2014.8314 & EFRVYSSANNCT Man1 & Inf \\
\hline & 13.4 & 2176.8842 & EFRYYSSANNCT Man2 & Inf \\
\hline N165 & 13.4 & 2338.937 & EFRUYSSANNCT Man 3 & Inf \\
\hline & 13.4 & 2500.9898 & EFRYYSSANNCT Man4 & Inf \\
\hline & 13.4 & 2987.1482 & EFRVYSSANNCT Man7 & Inf \\
\hline & 13.4 & 3149.201 & EFRYYSSANNCT ManB & Inf \\
\hline & 13.4 & 3311.2538 & EFRVYSSANNCT Man9 & Int \\
\hline & 13.446 & 2663.0426 & EFRYYSSANNCT Man5 & obs \\
\hline & 19.8 & 3001.1428 & YSSANNCTFEYVS G1 & $\operatorname{lnf}$ \\
\hline & 19.852 & 3147.2007 & YSSANNCTFEYVS G1F & obs \\
\hline & 20.2 & 2839.09 & YSSANNCTFEYVS GO & Int \\
\hline & 20.2 & 2108.8256 & YSSANNCTFEYVS Man1 & Inf \\
\hline & 20.2 & 2270.8784 & YSSANNCTFEYVS Man2 & Inf \\
\hline & 20.2 & 2432.9312 & YSSANNCTFEYVS Man3 & Inf \\
\hline N165 & 20.2 & 2594.984 & YSSANNCTFEYVS Man4 & $\operatorname{lnf}$ \\
\hline & 20.2 & 2757.0368 & YSSANNCTFEYVS ManS & Inf \\
\hline & 20.2 & 2919.0896 & YSSANNCTFEYVS Man6 & Inf \\
\hline & 20.2 & 3081.1424 & YSSANNCTFEYUS Man? & Inf \\
\hline & 20.2 & 3243.1952 & YSSANNCTFEYYS Man8 & Inf \\
\hline & 20.2 & 3405.248 & YSSANNCTFEYVS Man9 & Inf \\
\hline & 20.29 & 2985.1479 & YSSANNCTFEYVS GOF & $\operatorname{lnf}$ \\
\hline & 20.61 & 2744.1259 & GP1+Hex2 & Inf \\
\hline$G P 1$ & 20.926 & 2780.0707 & $G P 1+360.1$ & Obs \\
\hline$G P_{1}$ & 21.144 & 2420.0129 & GP1 & obs \\
\hline & 21.41 & 2456.9655 & $6 P 1+37.0$ & obs \\
\hline & 20.8 & 2676.0267 & SSANNCTFEYVS GO & $\operatorname{Inf}$ \\
\hline & 20.8 & 1945.7623 & SSANNCTFEYVS Man1 & Int \\
\hline & 20.8 & 2107.8151 & SSANNCTFEYVS Man2 & Inf \\
\hline & 20.8 & 2269.8679 & SSANNCTFEYVS Man3 & Int \\
\hline & 20.8 & 2431.9207 & SSANNCTFEYUS Man4 & Inf \\
\hline N165 & 20.8 & 2593.9735 & SSANNCTFEYVS ManS & Inf \\
\hline & 20.8 & 2756.0263 & SSANNCTFEYVS Man6 & Inf \\
\hline & 20.8 & 2918.0791 & SSANNCTFEYVS Man7 & Int \\
\hline & 20.8 & 3080.1319 & SSANNCTFEYVS ManB & Inf \\
\hline & 20.8 & 3242.1847 & SSANNCTFEYVS Man9 & Inf \\
\hline & 20.881 & 2822.0846 & SSANNCTFEYYS GOF & Obs \\
\hline & 20.9 & 2457.9939 & QDVNCTEVPV GO & Inf \\
\hline & 20.9 & 1727.7295 & QDVNCTEVPV Man1 & Inf \\
\hline & 20.9 & 1889.7823 & QDVNCTEVPV Man2 & Inf \\
\hline & 20.9 & 2051.8351 & QDVNCTEVPV Man3 & Inf \\
\hline & 20.9 & 2213.8879 & QDVNCTEVPV ManA & Inf \\
\hline N616 & 20.9 & 2375.9407 & QDVNCTEVPV Man5 & Inf \\
\hline & 20.9 & 2537.9935 & QDVNCTEVPV Man6 & Inf \\
\hline & 20.9 & 2700.0463 & QDVNCTEVPV Man? & Inf \\
\hline & 20.9 & 2862.0991 & QDVNCTEVPV Man 8 & Inf \\
\hline & 20.9 & 3024.1519 & QDVNCTEVPV Man? & Inf \\
\hline & 20.982 & 2604.0518 & QDVNCTEVPV GOF & Obs \\
\hline & 21.2 & 2677.0431 & NENGIITDAVDCA GO & Inf \\
\hline & 21.2 & 1946.7787 & NENGTITDAVDCAMan1 & Inf \\
\hline & 21.2 & 2108.8315 & NENGIITDAVDCA Man2 & Inf \\
\hline & 21.2 & 2270.8843 & NENGTITDAVDCA Man3 & Int \\
\hline & 21.2 & 2432.9371 & NENGTITDAVDCA Man4 & Inf \\
\hline N282 & 21.2 & 2594.9899 & NENGTIIDAVDCA Man5 & $\operatorname{lnf}$ \\
\hline & 21.2 & 2757.0427 & NENGTITDAVDCA Man6 & Inf \\
\hline & 21.2 & 2919.0955 & NENGTITDAVDCA Man7 & Inf \\
\hline & 21.2 & 3081.1483 & NENGTIIDAVDCA Man8 & Inf \\
\hline & 21.2 & 3243.2011 & NENGTITDAVDCA Man9 & Inf \\
\hline & 21.236 & 2823.101 & NENGIITDAVDCA GOF & Obs \\
\hline & 21.7 & 3255.2204 & NCTFEYUSQPF Man9 & Inf \\
\hline & 21.758 & 3093.1676 & NCTFEYVSQPF Man8 & Obs \\
\hline & 22 & 2689.0624 & NCTFEYVSOPF GO & $\operatorname{lnf}$ \\
\hline & 22 & 2835.1203 & NCTFEYVSQPF GOF & Inf \\
\hline & 22 & 1958.798 & NCTFEYVSQPF Man1 & Inf \\
\hline N165 & 22 & 2120.8508 & NCTFEYVSQPF Man2 & $\operatorname{lnf}$ \\
\hline & 22 & 2282.9036 & NCTFEYVSQPF Man3 & Int \\
\hline & 22 & 2444.9564 & NCTFEYVSQPF Man4 & Inf \\
\hline & 22 & 2607.0092 & NCTFEYUSQPF ManS & Inf \\
\hline & 22 & 2769.062 & NCTFEYVSQPF Man6 & Inf \\
\hline & 22.044 & 2931.1148 & NCTFEYVSQPF Man7 & Obs \\
\hline
\end{tabular}

\begin{tabular}{|c|c|c|c|c|}
\hline $\begin{array}{l}\text { Glyean } \\
\text { posn. }\end{array}$ & RT $(\min )$ & Mass & Glycopeptide & $\begin{array}{c}\text { Observed/ } \\
\text { Inferred }\end{array}$ \\
\hline \multirow{32}{*}{ N343 } & 23.295 & 2438.9204 & GEVFNAT Man8 & Obs \\
\hline & 23.399 & 2838.1073 & GEVFNAT Complex NeuAC (F)2 & obs \\
\hline & 23.762 & 2276.8676 & GEVFNAT Man7 & obs \\
\hline & 23.8 & 2600.9732 & GEVFNAT Man9 & $\operatorname{lnf}$ \\
\hline & 23.94 & 2692.0536 & GEVFNAT Complex NeUAC F & obs \\
\hline & 23.962 & 2114.8148 & GEVFNAT Man6 & obs \\
\hline & 24.203 & 1628.6564 & GEVFNAT Man3 & obs \\
\hline & 24.206 & 2488.9838 & GEVFNAT G1/FI2 & Obs \\
\hline & 24.272 & 2504.9787 & GEVFNAT G2F & obs \\
\hline & 24.3 & 1304.5508 & GEVFNAT Main1 & Int \\
\hline & 24.3 & 1466.6036 & GEVFNAT Man2 & Inf \\
\hline & 24.349 & 1790.7092 & GEVFNAT Man4 & obs \\
\hline & 24.352 & 1952.762 & GEVFNAT Man5 & obs \\
\hline & 24.618 & 2383.9525 & GEVFNAT GOF+GleNAC & obs \\
\hline & 24.7 & 2196.868 & GEVFNAT G1 & Int \\
\hline & 24.7 & 2358.9208 & GEVFNAT G2 & Int \\
\hline & 24.713 & 2342.9259 & GEVFNAT G1F & Obs \\
\hline & 24.9 & 2034.8152 & GEVFNAT GO & Inf \\
\hline & 24.967 & 2180.8731 & GEVFNAT GOF & obs \\
\hline & 25 & 2237.8946 & GEVFNAT GO+GIcNAC & Inf \\
\hline & 25.323 & 2383.9525 & GEVFNAT GOF+GICNAC & obs \\
\hline & 25.4 & 2650.0162 & GEVFNAT A1 & Int \\
\hline & 25.4 & 2941.1116 & GEVFNAT A2 & Inf \\
\hline & 25.476 & 2983.1586 & GEVFNAT A1(F)2-Gal*GICNAC & obs \\
\hline & 25.492 & 2796.0741 & GEVFNAT A1F & obs \\
\hline & 25.734 & 2837.1007 & GEVFNAT A1F-Gal+GICNAC & obs \\
\hline & 25.771 & 3087.1695 & GEVFNAT A2F & obs \\
\hline & 25.9 & 1142.49791 & GEVFNAT (GlcNAC)? & Int \\
\hline & 25.9 & 939.4186 & GEVFNAT GlcNAc stump & Int \\
\hline & 25.9555 & 736.33917 & GEVFNAT peptide & obs \\
\hline & 26.023 & 2634.0213 & GEVFNAT A1F-Gal & obs \\
\hline & 26.161 & 3109.1451 & GEVFNAT Very Complex & obs \\
\hline \multirow{2}{*}{ GP2 } & 25.198 & 1545.693 & GP2 & obs \\
\hline & 25.587 & 2173.9303 & $G P 2+628.0$ & Obs \\
\hline & 25.2 & 2123.8516 & (G)EVFNAT GOF & $\ln f$ \\
\hline & 25.2 & 1895.7405 & (G)EVFNAT ManS & Int \\
\hline N343 & 25.203 & 1977.7937 & (G)EVFNAT GO & obs \\
\hline 10345 & 26.5 & 1933.7675 & GEVFNA(T) GO & Int \\
\hline & 26.5 & 2079.8254 & GEVFNA(T) GOF & Inf \\
\hline & 26.566 & 1851.7143 & GEVFNA(T) Man5 & obs \\
\hline & 27.039 & 3295.3341 & GGVSVITPGINISNQ Man9 & obs \\
\hline & 27.6 & 2729.1761 & GGVSVITPGTNTSNQ GO & $\ln f$ \\
\hline & 27.6 & 2875.234 & GGVSVITPGTNTSNQ GOF & Inf \\
\hline & 27.6 & 1998.9117 & GGVSVITPGINTSNQ Man1 & Int \\
\hline & 27.6 & 2160.9645 & GGVSVITPGTNTSNQ Man2 & $\ln t$ \\
\hline N603 & 27,6 & 2323.0173 & GGVSVIIPGINISNQ Man3 & Int \\
\hline & 27.6 & 2485.0701 & GGVSVITPGINISNQ Man 4 & Int \\
\hline & 27.6 & 2647.1229 & GGVSVITPGTNTSNQ ManS & $\operatorname{Inf}$ \\
\hline & 27.6 & 2809.1757 & GGVSVITPGTNTSNQ Man6 & Inf \\
\hline & 27.6 & 2971.2285 & GGVSVITPGINTSNQ Man7 & Inf \\
\hline & 27.627 & 3133.2813 & GGVSVITPGINISNQ Man8 & Obs \\
\hline & 27.7 & 3092.2109 & MESEFRVYSSANNCT GO & Inf \\
\hline & 27.7 & 3238.2688 & MESEFRVYSSANNCT GOF & Int \\
\hline & 27.7 & 2361.9465 & MESEFAVYSSANNCT Man1 & $\ln f$ \\
\hline & 27.7 & 2523.9993 & MESEFRVYSSANNCT Man2 & Inf \\
\hline & 27.7 & 2686.0521 & MESEFRVYSSANNCT Man3 & Inf \\
\hline N165 & 27.7 & 2848.1049 & MESEFAVYSSANNCT Man4 & Inf \\
\hline & 27.7 & 3172.2105 & MESEFRVYSSANNCT Man6 & Inf \\
\hline & 27.7 & 3334.2633 & MESEFRVYSSANNCT Man7 & Inf \\
\hline & 27.7 & 3496.3161 & MESEFAVYSSANNCT Man8 & Inf \\
\hline & 27.7 & 3658.3689 & MESEFRVYSSANNCT Man9 & $\operatorname{Inf}$ \\
\hline & 27.778 & 3010.1577 & MESEFRWYSSANNCT ManS & obs \\
\hline GP3 & 27.785 & 2971.2343 & GP3+GICNAC & obs \\
\hline & 28.022 & 2768.1567 & GP3 & Obs \\
\hline & 29.861 & 2703.1208 & PPIKDFGGFNFS Man6 & obs \\
\hline & 30.5 & 2623.1212 & PPIKDFGGFNFS GO & $\ln f$ \\
\hline & 30.5 & 2769.1791 & PPIKDFGGFNFS GOF & Int \\
\hline & 30.5 & 2054.9096 & PPIKDFGGFNFS Man2 & Inf \\
\hline & 30.5 & 2216.9624 & PPIKDFGGFNFS Man3 & Inf \\
\hline NoO1 & 30.5 & 2379.0152 & PPIKDFGGFNFS Man4 & $\operatorname{lnf}$ \\
\hline & 30.5 & 2541.068 & PPIKDFGGFNFS ManS & Inf \\
\hline & 30.5 & 2865.1736 & PPIKDFGGFNF5 Man7 & Int \\
\hline & 30.5 & 3027.2264 & PPIKDFGGFNF5 Man8 & Int \\
\hline & 30.5 & 3189.2792 & PPIKDFGGFNFS Man9 & Int \\
\hline & 31.037 & 2703.1208 & PPIKDFGGFNFS Man6 & Obs \\
\hline & 31.2 & 2206.9363 & RFPNIT G1 & $\operatorname{lnf}$ \\
\hline & 31.204 & 2352.9942 & RFPNIT G1F & Obs \\
\hline & 32 & 2044.8835 & RFPNIT GO & $\operatorname{linf}$ \\
\hline & 32 & 1314.6191 & RFPNIT Man1 & $\ln f$ \\
\hline & 32 & 1476.6719 & RFPNIT Man2 & int \\
\hline & 32 & 1638.7247 & RFPNIT Man3 & Int \\
\hline N331 & 32 & 1800.7775 & RFPNIT Man4 & Inf \\
\hline & 32 & 1962.8303 & RFPNIT ManS & Int \\
\hline & 32 & 2124.8831 & RFPNIT Man6 & Inf \\
\hline & 32 & 2286.9359 & RFPNIT Man7 & $\ln f$ \\
\hline & 32 & 2448.9887 & RFPNIT ManB & $\operatorname{Inf}$ \\
\hline & 32 & 2611.0415 & RFPNIT Man9 & Inf \\
\hline & 32.044 & 2190.9414 & RFPNIT GOF & Obs \\
\hline
\end{tabular}


bioRxiv preprint doi: https://doi.org/10.1101/2020.07.24.217562; this version posted July 27, 2020. The copyright holder for this preprint (which was not certified by peer review) is the author/funder, who has granted bioRxiv a license to display the preprint in perpetuity. It is made available under aCC-BY-ND 4.0 International license.

Table 1b. Spike elastase glycopeptide mass retention time database (PCDL) containing data for 140 observed glycopeptides and data for a further 306 inferred glycopeptides (RT 32-60 min and key)

\begin{tabular}{|c|c|c|c|c|}
\hline $\begin{array}{l}\text { Glycan } \\
\text { posn. }\end{array}$ & RT (min) & Mass & Glycopeptide & $\begin{array}{c}\text { Observed/ } \\
\text { Inferred }\end{array}$ \\
\hline \multirow{11}{*}{ N149 } & 32.4 & 3333.3262 & VYYHKNNNSWM Man9 & Inf \\
\hline & 32.463 & 2847.1678 & VYYHKNNKSWM Man6 & Obs \\
\hline & 32.463 & 3171.2734 & VYYHKNNKSWM Man8 & obs \\
\hline & 32.6 & 2767.1682 & VYYHKNNNSWM G0 & Inf \\
\hline & 32.6 & 2913.2261 & VYYHKNNKSWM GOF & Inf \\
\hline & 32.6 & 2036.9038 & VTYHKNNKSWM Man1 & Inf \\
\hline & 32.6 & 2198.9566 & VTrHKNNNKSWM Man2 & Inf \\
\hline & 32.6 & 2361.0094 & VYYHKNNKSWM Man3 & Inf \\
\hline & 32.6 & 2523.0622 & VYYHKNNKSWM Man4 & Inf \\
\hline & 32.6 & 2685.115 & VrYHKNNKSWM ManS & Inf \\
\hline & 32.682 & 3009.2206 & VYYHKNNKSWM Man7 & Obs \\
\hline \multirow{3}{*}{ GP4 } & 33.611 & 2694.1185 & GP4 Man6 & Obs \\
\hline & 33.8 & 2760.1768 & GP4 GOF & Inf \\
\hline & 33.819 & 2532.0657 & GP4 Mans & obs \\
\hline \multirow{11}{*}{ N17 } & 32.463 & 2828.1148 & CVNLTTRT Man9 & Obs \\
\hline & 33.8 & 2261.9568 & CVNLTTRT GO & Inf \\
\hline & 33.8 & 2408.0147 & CVNLITRT GOF & Inf \\
\hline & 33.8 & 1531.6924 & CVNLTTRT Man1 & Inf \\
\hline & 33.8 & 1693.7452 & CVNLTTRT Man2 & Inf \\
\hline & 33.8 & 1855.798 & CVNLTTRT Man3 & Inf \\
\hline & 33.8 & 2017.8508 & CVNLTTRT Man4 & Inf \\
\hline & 33.8 & 2179.9036 & CVNLTTRT ManS & Inf \\
\hline & 33.8 & 2341.9564 & CVNLTTRT Man6 & Inf \\
\hline & 33.8 & 2504.0092 & CVNLTTRT Man7 & Inf \\
\hline & 33.841 & 2666.062 & CVNLTTRT Man8 & Obs \\
\hline & 34.6 & 1848.7511 & VFNAT GO & Inf \\
\hline & 34.6 & 1994.809 & VFNAT GOF & Inf \\
\hline & 34.6 & 1118.4867 & VFNAT Man1 & Inf \\
\hline & 34.6 & 1280.5395 & VFNAT Man2 & Inf \\
\hline & 34.6 & 1442.5923 & VFNAT Man3 & Inf \\
\hline N343 & 34.6 & 2252.8563 & VFNAT Man8 & Inf \\
\hline & 34.6 & 2414.9091 & VFNAT Man9 & Inf \\
\hline & 34.656 & 1928.7507 & VFNAT Man6 & Obs \\
\hline & 34.674 & 2090.8035 & VFNAT Man7 & Obs \\
\hline & 34.682 & 1766.6979 & VFNAT ManS & Obs \\
\hline & 34.688 & 1604.6451 & VFNAT Man4 & Obs \\
\hline & 35.101 & 2195.8349 & NFTI Man8 & Obs \\
\hline & 35.353 & 1709.6765 & NFTI Mans & Obs \\
\hline & 35.373 & 1385.5709 & NFII Man3 & Obs \\
\hline & 35.374 & 2033.7821 & NFTI Man7 & Obs \\
\hline & 35.8 & 2357.8877 & NFTI Man9 & Inf \\
\hline & 35.86 & 1871.7293 & NFTI Man6 & Obs \\
\hline & 36.2 & 2406.9307 & NFTI A1 & Inf \\
\hline & 36.2 & 2552.9886 & NFTI A1F & Inf \\
\hline & 36.2 & 2698.0261 & NFT1 A2 & Inf \\
\hline & 36.2 & 2844.084 & NFTI A2F & Inf \\
\hline N717 & 36.2 & 1791.7297 & NFTI GO & Inf \\
\hline N717 & 36.2 & 1994.8091 & NFTI GO •GlcNAC & Obs \\
\hline & 36.2 & 2140.867 & NFTI GOF +GlCNAC & Obs \\
\hline & 36.2 & 1953.7825 & NFTI G1 & Inf \\
\hline & 36.2 & 2115.8353 & NFTIG2 & Inf \\
\hline & 36.2 & 2261.8932 & NFTI G2F & Inf \\
\hline & 36.2 & 1061.4653 & NFTI Man1 & Inf \\
\hline & 36.2 & 1223.5181 & NFTI Man2 & Inf \\
\hline & 36.295 & 1547.6237 & NFTI Man4 & Obs \\
\hline & 36.599 & 2099.8404 & NFTI G1F & Obs \\
\hline & 36.77 & 2089.8308 & NFTI ManS+380.2 & Obs \\
\hline & 36.896 & 1937.7876 & NFTI GOF & Obs \\
\hline & 35.4 & 2765.11 & PFFSNVTW G2F & Inf \\
\hline & 35.4 & 1564.6821 & PFFSNVTW Man1 & Inf \\
\hline & 35.4 & 1726.7349 & PFFSNVTW Man2 & Inf \\
\hline & 35.4 & 1898.7877 & PFFSNVITW Man 3 & Inf \\
\hline & 35.4 & 2050.8405 & PFFSNVTTW Man4 & Inf \\
\hline & 35.4 & 2212.8933 & PFFSNVITW ManS & Inf \\
\hline & 35.4 & 2536.9989 & PFFSNVTWW Man7 & Inf \\
\hline & 35.4 & 2699.0517 & PFFSNVTWW Man8 & Inf \\
\hline N61 & 35.4 & 2861.1045 & PFFSNVTWW Man9 & Inf \\
\hline & 35.437 & 2619.0521 & PFFSNVTW G2 & Obs \\
\hline & 35.601 & 2456.9993 & PFFSNVTW G1 & Obs \\
\hline & 36 & 2498.0259 & PFFSNVTWW GO +GleNAC & Inf \\
\hline & 36 & 2441.0044 & PFFSNVITW GOF & Inf \\
\hline & 36 & 2644.0838 & PFFSNNVTW GOF + GlcNAC & Inf \\
\hline & 36 & 2603.0572 & PFFSNNTW G1F & Int \\
\hline & 36.025 & 2294.9465 & PFFSNVTW GO & obs \\
\hline & 36.854 & 2374.9461 & PFFSNVTWW Man6 & Obs \\
\hline & 36.5 & 2181.8836 & FGEVFNAT GO & Inf \\
\hline & 36.5 & 2327.9415 & FGEVFNAT GOF & Inf \\
\hline & 36.5 & 1451.6192 & FGEVFNAT Man1 & Inf \\
\hline & 36.5 & 1613.672 & FGEVFNAT Man2 & Inf \\
\hline & 36.5 & 1775.7248 & FGEVFNAT Man3 & Inf \\
\hline N343 & 36.5 & 1937.7776 & FGEVFNAT ManA & Inf \\
\hline & 36.5 & 2261.8832 & FGEVFNAT Man6 & Inf \\
\hline & 36.5 & 2423.936 & FGEVFNAT Man7 & Inf \\
\hline & 36.5 & 2585.9888 & FGEVFNAT Man8 & Inf \\
\hline & 36.5 & 2748.0416 & FGEVFNAT Man9 & Inf \\
\hline & 36.528 & 2099.8304 & FGEVFNAT Man5 & Obs \\
\hline
\end{tabular}

\begin{tabular}{|c|c|c|}
\hline $\begin{array}{l}\text { Glycan } \\
\text { posn. }\end{array}$ & RT $(\min )$ & Mass \\
\hline & 36.8 & 2552.0511 \\
\hline & 36.8 & 2698.109 \\
\hline & 36.8 & 1821.7867 \\
\hline & 36.8 & 1983.8395 \\
\hline & 36.8 & 2145.8923 \\
\hline N343 & 36.8 & 2307.9451 \\
\hline & 36.8 & 2469.9979 \\
\hline & 36.8 & 2794.1035 \\
\hline & 36.8 & 2956.1563 \\
\hline & 36.8 & 3118.2091 \\
\hline & 36.864 & 2632.0507 \\
\hline GP6 & 40.948 & 1570.7208 \\
\hline & 41.645 & 1881.7363 \\
\hline & 42.6 & 1855.7569 \\
\hline & 42.6 & 2001.8148 \\
\hline & 42.6 & 1125.4925 \\
\hline & 42.6 & 1287.5453 \\
\hline & 42.6 & 1611.6509 \\
\hline N331 & 42.6 & 1773.7037 \\
\hline & 42.6 & 1935.7565 \\
\hline & 42.6 & 2097.8093 \\
\hline & 42.6 & 2259.8621 \\
\hline & 42.6 & 2421.9149 \\
\hline & 42.631 & 1449.5981 \\
\hline & 46.236 & 3854.7283 \\
\hline & 46.369 & 3788.67 \\
\hline & 46.424 & 3626.6226 \\
\hline & 46.5 & 3546.6176 \\
\hline & 46.5 & 3692.6755 \\
\hline & 46.5 & 3708.6704 \\
\hline & 46.5 & 3950.7228 \\
\hline & 46.506 & 3464.5644 \\
\hline & 46.506 & 3626.6172 \\
\hline & 46.509 & 3530.6227 \\
\hline & 46.509 & 3733.7021 \\
\hline N801 & 46.538 & 3140.4588 \\
\hline & 46.568 & 2978.406 \\
\hline & 46.575 & 2654.3004 \\
\hline & 46.581 & 2816.3532 \\
\hline & 46.601 & 3302.5116 \\
\hline & 46.668 & 3384.5648 \\
\hline & 46.966 & 3587.6442 \\
\hline & 47.5 & 4145.8237 \\
\hline & 47.5 & 4290.8612 \\
\hline & 47.5 & 4436.9191 \\
\hline & 48.492 & 3999.7658 \\
\hline & 48.1 & 3630.5282 \\
\hline & 48.1 & 3776.5861 \\
\hline & 48.1 & 3921.6236 \\
\hline & 48.1 & 4067.6815 \\
\hline & 48.121 & 3485.4907 \\
\hline & 48.27 & 4145.822 \\
\hline & 48.332 & 3419.4324 \\
\hline & 48.339 & 3323.4379 \\
\hline & 48.408 & 3257.3796 \\
\hline & 48.453 & 3364.4645 \\
\hline & 48.462 & 3161.3851 \\
\hline & 48.499 & 3983.7666 \\
\hline non & 48.518 & 3095.3268 \\
\hline N801 & 48.6 & 1716.8512 \\
\hline & 48.6 & 2123.01 \\
\hline & 48.6 & 3015.3272 \\
\hline & 48.6 & 3177.38 \\
\hline & 48.6 & 3339.4328 \\
\hline & 48.6 & 1919.9306 \\
\hline & 48.6 & 2285.0628 \\
\hline & 48.6 & 2447.1156 \\
\hline & 48.6 & 2609.1684 \\
\hline & 48.6 & 2771.2212 \\
\hline & 48.6 & 3581.4852 \\
\hline & 48.623 & 3218.4066 \\
\hline & 48.647 & 2933.274 \\
\hline & 50.406 & 3614.5291 \\
\hline & 51.783 & 2908.1464 \\
\hline & 51.942 & 3136.2575 \\
\hline & 52.073 & 2131.0262 \\
\hline & 52.167 & 2421.988 \\
\hline & 52.185 & 2259.9352 \\
\hline & 52.229 & 2666.094 \\
\hline & 52.265 & 2746.0936 \\
\hline & 52.3 & 3281.295 \\
\hline & 52.3 & 3572.3904 \\
\hline & 52.3 & 2828.1468 \\
\hline N343 & 52.3 & 2990.1996 \\
\hline & 52.32 & 2974.2047 \\
\hline & 52.445 & 3015.2313 \\
\hline & 52.5 & 1935.8296 \\
\hline & 52.5 & 2097.8824 \\
\hline & 52.5 & 3070.1992 \\
\hline & 52.5 & 3232.252 \\
\hline & 52.575 & 2584.0408 \\
\hline & 52.632 & 2812.1519 \\
\hline & 53.304 & 3427.3529 \\
\hline & 53.961 & 3718.4483 \\
\hline & 60.483 & 1861.7463 \\
\hline
\end{tabular}

\begin{tabular}{|c|c|}
\hline \multicolumn{2}{|c|}{ Key to Glycan Structures } \\
\hline GlcNAc stump & - \\
\hline (GleNAC)2 & 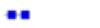 \\
\hline $\operatorname{Man} 1$ & 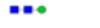 \\
\hline $\operatorname{Man} 2$ & M. \\
\hline Man3 & $\omega \bullet$ \\
\hline Man4 & 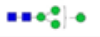 \\
\hline Mans & $=0 \%:$ \\
\hline Man6 & $m \div:$ \\
\hline $\operatorname{Man} 7$ & $=0 \%:$ \\
\hline Mans & $=<::$ \\
\hline Mang & $\cdots$ \\
\hline 6० & $\cdots \bullet: Z$ \\
\hline GO+GleNAC & $\cdots: B=$ \\
\hline GOF & in: : \\
\hline GOF+GleNAC & 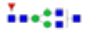 \\
\hline G1 & ๓-०: \\
\hline G1F & :-0:8 \\
\hline G1(F)2 & :-0:8) \\
\hline 62 & $\cdots: 8$ \\
\hline G2F & :..:: \\
\hline A1 & $\cdots \bullet:$ \\
\hline A1F & ப.००:: \\
\hline A1F-Gal & ¿...:日 \\
\hline A1F-Gal+GicNAc & ¿as: =" \\
\hline$A 1(F) 2-G a l+G l c N A C$ & 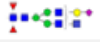 \\
\hline$A 2$ & *०:: \\
\hline A2F & 1.0.:: : \\
\hline
\end{tabular}

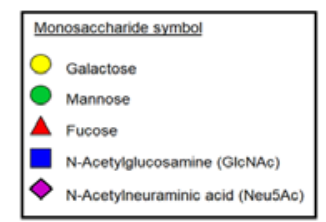


bioRxiv preprint doi: https://doi.org/10.1101/2020.07.24.217562; this version posted July 27,2020 . The copyright holder for this preprint (which was not certified by peer review) is the author/funder, who has granted bioRxiv a license to display the preprint in perpetuity. It is made available under aCC-BY-ND 4.0 International license.

The complete Spike PCDL database is available to download in .cdb or .xlsx format here: https://zenodo.org/record/3958218\#.Xxn BChKhoY

Figure 3. Combined Extracted Ion Chromatogram (EIC) for 27 isoforms of glycopeptide GEVFNAT (N343) within +/- 2 min retention time window from RBD. Only three glycans are labelled, the remainder are listed in the accompanying table 2, below.

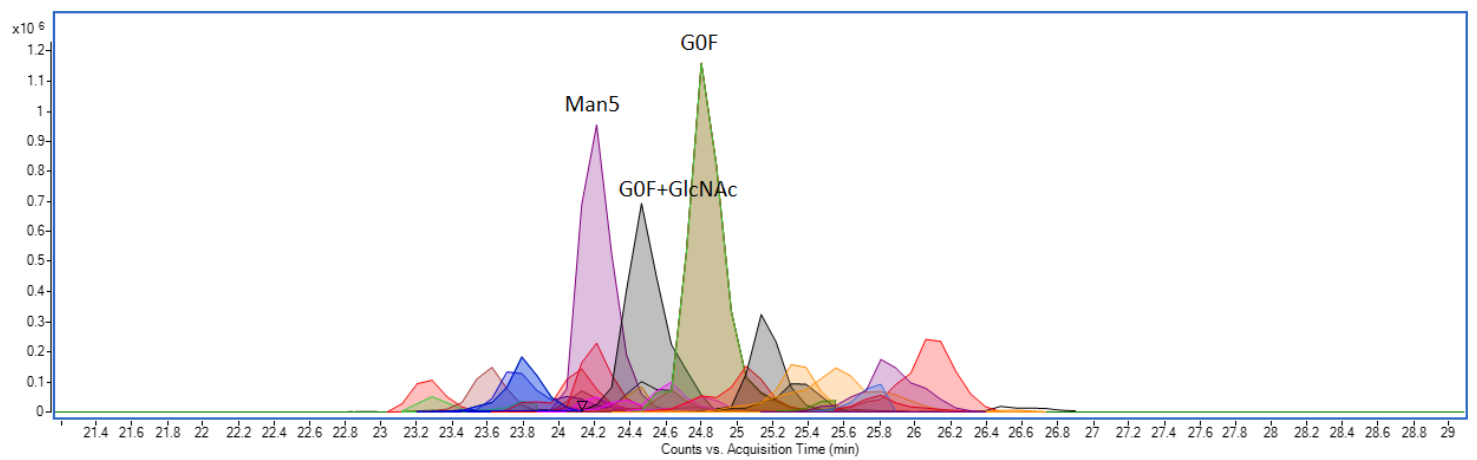

Table 2. GEVFNAT glycopeptide (N343) isoforms from RBD shown in Figure 3

\begin{tabular}{|c|c|c|c|c|c|c|c|c|c|}
\hline Name & Mass & RT & Volume & ppm error & Name & Mass & RT & Volume & ppm error \\
\hline GEVFNAT Complex NeuAc (F)2 & 2838.1078 & 23.26 & 733674 & -0.2 & GEVFNAT G1F & 2342.9180 & 24.62 & 1090359 & 3.4 \\
\hline GEVFNAT Man8 & 2438.9113 & 23.30 & 744169 & 3.7 & GEVFNAT G0 & 2034.8089 & 24.80 & 950868 & 3.1 \\
\hline GEVFNAT Man7 & 2276.8615 & 23.61 & 1867525 & 2.7 & GEVFNAT GOF & 2180.8688 & 24.82 & 12980259 & 2.0 \\
\hline GEVFNAT Complex NeUAC F & 2692.0523 & 23.77 & 1266219 & 0.5 & GEVFNAT A1(F)2-Gal+GIcNAC & 2983.1427 & 24.91 & 732781 & 5.3 \\
\hline GEVFNAT Man6 & 2114.8091 & 23.80 & 2432950 & 2.7 & (G)EVFNAT G0 & 1977.7887 & 25.05 & 2947447 & 2.5 \\
\hline GEVFNAT G1(F)2 & 2488.9768 & 24.09 & 1292274 & 2.8 & GEVFNAT GOF+GIcNAC & 2383.9464 & 25.16 & 3836871 & 2.6 \\
\hline GEVFNAT G2F & 2504.9755 & 24.14 & 1007345 & 1.3 & GEVFNAT A1F & 2796.0641 & 25.33 & 1512501 & 3.6 \\
\hline GEVFNAT Man4 & 1790.7051 & 24.20 & 3040952 & 2.3 & GEVFNAT A1(F)2-Gal+GIcNAC & 2983.1444 & 25.34 & 1320994 & 4.8 \\
\hline GEVFNAT Man5 & 1952.7583 & 24.20 & 12492713 & 1.9 & GEVFNAT A1F-Gal & 2634.0105 & 25.51 & 608358 & 4.1 \\
\hline GEVFNAT Man3 & 1628.6504 & 24.20 & 666739 & 3.7 & GEVFNAT A2F & 3087.1629 & 25.77 & 805142 & 2.2 \\
\hline GEVFNAT G1F & 2342.9183 & 24.37 & 1333546 & 3.3 & GEVFNAT A1F & 2796.0644 & 25.80 & 541989 & 3.5 \\
\hline GEVFNAT GOF & 2180.8638 & 24.45 & 953034 & 4.3 & GEVFNAT A1F-Gal & 2634.0125 & 25.86 & 3692097 & 3.4 \\
\hline GEVFNAT GOF+GICNAC & 2383.9436 & 24.47 & 9038631 & 3.7 & & & & & \\
\hline
\end{tabular}

Figure 4 illustrates a complete glycan fragmentation series for RBD glycopeptide GEVFNAT-Man5 showing the peptide stump (GEVFNAT-GICNAC) and mannose ladders. Calculated mass errors are shown in table 3.

Figure 4. Complete glycan fragmentation series for RBD glycopeptide GEVFNAT-Man5 (N343)

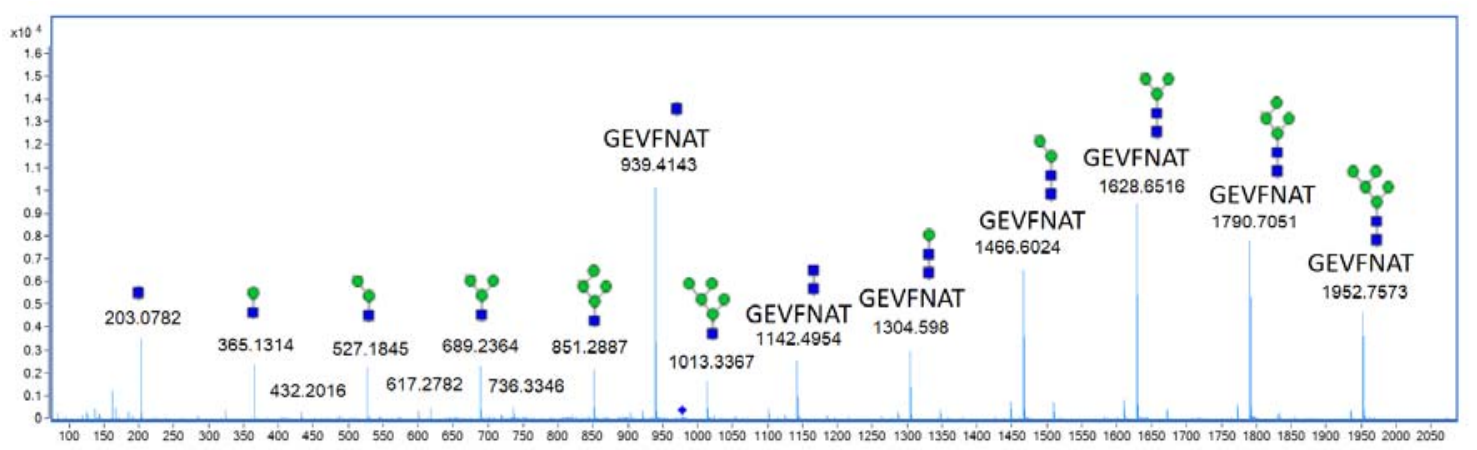

Deconvoluted Mass 
bioRxiv preprint doi: https://doi.org/10.1101/2020.07.24.217562; this version posted July 27,2020 . The copyright holder for this preprint (which was not certified by peer review) is the author/funder, who has granted bioRxiv a license to display the preprint in perpetuity. It is made available under aCC-BY-ND 4.0 International license.

Table 3. Glycan assignment and mass errors (parts per million) for RDB glycopeptide GEVFNAT-Man5

\begin{tabular}{|c|c|c|c|c|}
\hline $\begin{array}{l}\text { Deconvoluted } \\
\text { Mass Obs }\end{array}$ & Formula & Mass Calc & $\mathrm{ppm}$ & Assignment \\
\hline 203.0782 & $\mathrm{C}_{8} \mathrm{H}_{13} \mathrm{NO}_{5}$ & 203.0794 & -5.9 & GIcNAC \\
\hline 365.1314 & $\mathrm{C}_{8} \mathrm{H}_{13} \mathrm{~N} \mathrm{O}_{5}\left(\mathrm{C}_{6} \mathrm{H}_{10} \mathrm{O}_{5}\right) 1$ & 365.1322 & -2.2 & GlcNAc(Man)1 \\
\hline 527.1845 & $\mathrm{C}_{8} \mathrm{H}_{13} \mathrm{~N} \mathrm{O}_{5}\left(\mathrm{C}_{6} \mathrm{H}_{10} \mathrm{O}_{5}\right) 2$ & 527.1850 & -0.9 & GlcNAc(Man)2 \\
\hline 689.2364 & $\mathrm{C}_{8} \mathrm{H}_{13} \mathrm{~N} \mathrm{O}_{5}\left(\mathrm{C}_{6} \mathrm{H}_{10} \mathrm{O}_{5}\right)^{3}$ & 689.2364 & 0.0 & GlcNAc(Man)3 \\
\hline 851.2887 & $\mathrm{C}_{8} \mathrm{H}_{13} \mathrm{~N} \mathrm{O}_{5}\left(\mathrm{C}_{6} \mathrm{H}_{10} \mathrm{O}_{5}\right) 4$ & 851.2907 & -2.3 & GlcNAc(Man)4 \\
\hline 1013.3367 & $\mathrm{C}_{8} \mathrm{H}_{13} \mathrm{~N} \mathrm{O}_{5}\left(\mathrm{C}_{6} \mathrm{H}_{10} \mathrm{O}_{5}\right) 5$ & 1013.3435 & -6.7 & GlcNAc(Man)5 \\
\hline 939.4143 & $\mathrm{C}_{32} \mathrm{H}_{48} \mathrm{~N}_{8} \mathrm{O}_{12}\left(\mathrm{C}_{8} \mathrm{H}_{13} \mathrm{~N} \mathrm{O}_{5}\right)$ & 939.4185 & -4.5 & GEVFNAT (GICNAC) \\
\hline 1142.4954 & $\mathrm{C}_{32} \mathrm{H}_{48} \mathrm{~N}_{8} \mathrm{O}_{12}\left(\mathrm{C}_{8} \mathrm{H}_{13} \mathrm{NO}_{5}\right) 2$ & 1142.4979 & -2.2 & GEVFNAT (GICNAC) 2 \\
\hline 1304.5498 & $\mathrm{C}_{32} \mathrm{H}_{48} \mathrm{~N}_{8} \mathrm{O}_{12}\left(\mathrm{C}_{8} \mathrm{H}_{13} \mathrm{~N} \mathrm{O}_{5}\right) 2\left(\mathrm{C}_{6} \mathrm{H}_{10} \mathrm{O}_{5}\right) 1$ & 1304.5507 & -0.7 & GEVFNAT (GICNAC)2 (Man)1 \\
\hline 1466.6024 & $\mathrm{C}_{32} \mathrm{H}_{48} \mathrm{~N}_{8} \mathrm{O}_{12}\left(\mathrm{C}_{8} \mathrm{H}_{13} \mathrm{~N} \mathrm{O}_{5}\right) 2\left(\mathrm{C}_{6} \mathrm{H}_{10} \mathrm{O}_{5}\right) 2$ & 1466.6036 & -0.8 & GEVFNAT (GICNAC)2 (Man)2 \\
\hline 1628.6516 & $\mathrm{C}_{32} \mathrm{H}_{48} \mathrm{~N}_{3} \mathrm{O}_{12}\left(\mathrm{C}_{8} \mathrm{H}_{13} \mathrm{~N} \mathrm{O}_{5}\right) 2\left(\mathrm{C}_{6} \mathrm{H}_{10} \mathrm{O}_{5}\right) 3$ & 1628.6564 & -2.9 & GEVFNAT (GICNAC)2 (Man)3 \\
\hline 1790.7051 & $\mathrm{C}_{32} \mathrm{H}_{48} \mathrm{~N}_{8} \mathrm{O}_{12}\left(\mathrm{C}_{8} \mathrm{H}_{13} \mathrm{~N} \mathrm{O}_{5}\right) 2\left(\mathrm{C}_{6} \mathrm{H}_{10} \mathrm{O}_{5}\right) 4$ & 1790.7092 & -2.3 & GEVFNAT (GICNAC)2 (Man)4 \\
\hline 1952.7573 & $\mathrm{C}_{32} \mathrm{H}_{48} \mathrm{~N}_{8} \mathrm{O}_{12}\left(\mathrm{C}_{8} \mathrm{H}_{13} \mathrm{NO}_{5}\right) 2\left(\mathrm{C}_{6} \mathrm{H}_{10} \mathrm{O}_{5}\right) 5$ & 1952.7620 & -2.4 & GEVFNAT (GICNAC)2 (Man)5 \\
\hline
\end{tabular}

In the pseudo MS3 experiment glycans were lost by in-source decay. GEVFNAT-GIcNAc was isolated in the quadrupole and fragmented in the collision cell. Sequence confirmation for the peptide stump GEVFNAT-GICNAC is shown in Figure 5 with mass errors calculated in Table 4.

Figure 5. Pseudo MS3 fragmentation analysis of RBD glycopeptide stump GEVFNAT-GIcNAc (N343)

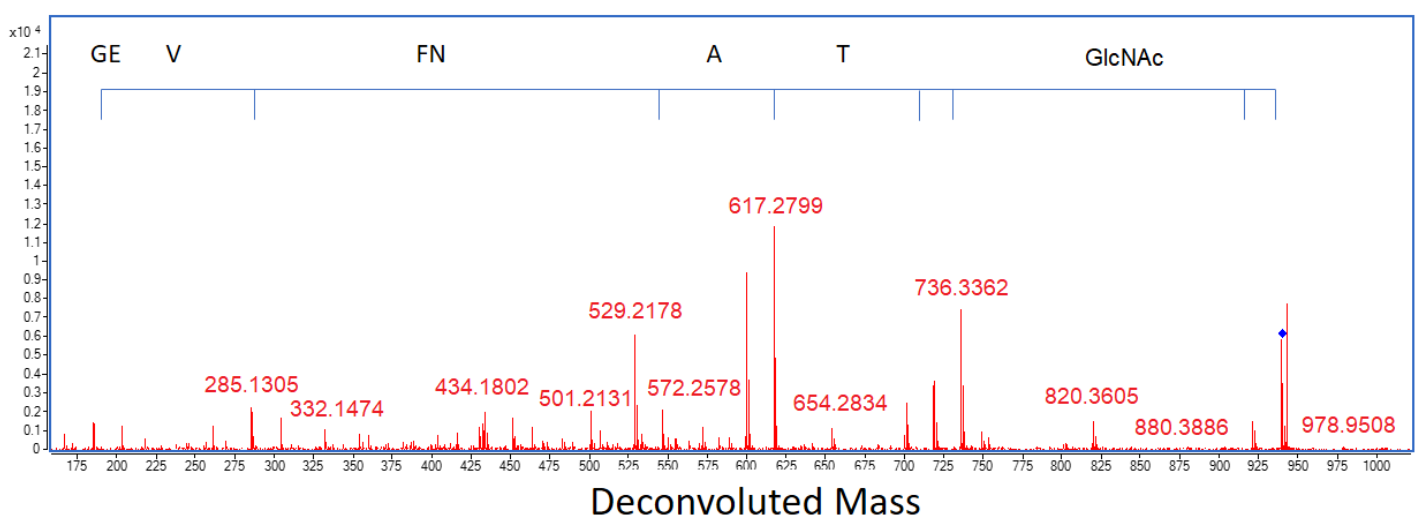

Table 4. Pseudo MS3 fragment ion assignment and mass errors (parts per million) for RBD glycopeptide stump GEVFNAT-GIcNAc (N343)

\begin{tabular}{|c|c|c|c|c|c|}
\hline $\begin{array}{l}\text { Deconvoluted } \\
\text { Observed Mass }\end{array}$ & Formula & $\begin{array}{c}\text { Calculated } \\
\text { Mass }\end{array}$ & $\begin{array}{l}\text { Error } \\
\text { (ppm) }\end{array}$ & $\begin{array}{l}\text { Fagment ion } \\
\text { assignment }\end{array}$ & $\begin{array}{l}\text { Peptide } \\
\text { Sequence }\end{array}$ \\
\hline 186.0645 & $\mathrm{C}_{7} \mathrm{H}_{10} \mathrm{~N}_{2} \mathrm{O}_{4}$ & 186.0641 & 2.1 & b2 & GE \\
\hline 285.1305 & $\mathrm{C}_{12} \mathrm{H}_{19} \mathrm{~N}_{3} \mathrm{O}_{5}$ & 285.1325 & -7.0 & b3 & GEV \\
\hline 546.2410 & $\mathrm{C}_{25} \mathrm{H}_{34} \mathrm{~N}_{6} \mathrm{O}_{8}$ & 546.2438 & -5.1 & b5 & GEVFN \\
\hline 617.2799 & $\mathrm{C}_{28} \mathrm{H}_{39} \mathrm{~N}_{7} \mathrm{O}_{9}$ & 617.2809 & -1.6 & b6 & GEVFNA \\
\hline 718.3257 & $\mathrm{C}_{32} \mathrm{H}_{46} \mathrm{~N}_{8} \mathrm{O}_{11}$ & 718.3286 & -4.0 & b7 & GEVFNAT \\
\hline 736.3362 & $\mathrm{C}_{32} \mathrm{H}_{48} \mathrm{~N}_{8} \mathrm{O}_{12}$ & 736.3392 & -4.1 & y7 & GEVFNAT \\
\hline 939.4174 & $\mathrm{C}_{32} \mathrm{H}_{48} \mathrm{~N}_{8} \mathrm{O}_{12} \mathrm{C}_{8} \mathrm{H}_{13} \mathrm{~N} \mathrm{O}_{5}$ & 939.4185 & -1.2 & M GlcNAc & GEVFNAT GICNAC \\
\hline 921.4064 & $\mathrm{C}_{32} \mathrm{H}_{46} \mathrm{~N}_{8} \mathrm{O}_{11} \mathrm{C}_{8} \mathrm{H}_{13} \mathrm{~N} \mathrm{O}_{5}$ & 921.408 & -1.7 & $\mathrm{M}$ GlcNAC $-\mathrm{H}_{2} \mathrm{O}$ & GEVFNAT GICNAC \\
\hline 820.3605 & $\mathrm{C}_{28} \mathrm{H}_{39} \mathrm{~N}_{7} \mathrm{O}_{9} \mathrm{C}_{8} \mathrm{H}_{13} \mathrm{~N} \mathrm{O}_{5}$ & 820.3603 & 0.2 & b6 $\mathrm{GlcNAC}-\mathrm{H}_{2} \mathrm{O}$ & GEVFNA GICNAC \\
\hline 749.3213 & $\mathrm{C}_{25} \mathrm{H}_{34} \mathrm{~N}_{6} \mathrm{O}_{8} \mathrm{C}_{8} \mathrm{H}_{13} \mathrm{~N} \mathrm{O}_{5}$ & 749.3232 & -2.5 & b5 GIcNAC $-\mathrm{H}_{2} \mathrm{O}$ & GEVFN GICNAC \\
\hline 701.3021 & $\mathrm{C}_{32} \mathrm{H}_{43} \mathrm{~N}_{7} \mathrm{O}_{11}$ & 701.3021 & 0.0 & b7 $-\mathrm{NH}_{3}$ & GEVFNAT \\
\hline 600.2529 & $\mathrm{C}_{28} \mathrm{H}_{36} \mathrm{~N}_{6} \mathrm{O}_{9}$ & 600.2544 & -2.5 & b6 $-\mathrm{NH}_{3}$ & GEVFNA \\
\hline 529.2178 & $\mathrm{C}_{25} \mathrm{H}_{31} \mathrm{~N}_{5} \mathrm{O}_{8}$ & 529.2173 & 0.9 & b5 $-\mathrm{NH}_{3}$ & GEVFN \\
\hline
\end{tabular}


Intact mass measurement of fully glycosylated Spike was unsuccessful due to the polydispersity of its innumerable glycoforms and the resulting dilution of ion signal. However, the smaller receptor binding domain, bearing only two glycosylation sites did prove amenable to intact mass analysis. Figure 6 shows twenty-one glycoforms for intact RBD, of which ten major glycoforms could be assigned. This showed that the principal glycan species were Man5, GOF and GOF+GlcNAc which was in agreement with the glycopeptide analysis.

Figure 6. Intact mass analysis of RBD showing the principal glycan species Man5, GOF and GOF+GICNAC in agreement with glycopeptide analysis. (This method cannot differentiate individual glycosylation sites, hence when two structures are possible, both are shown)

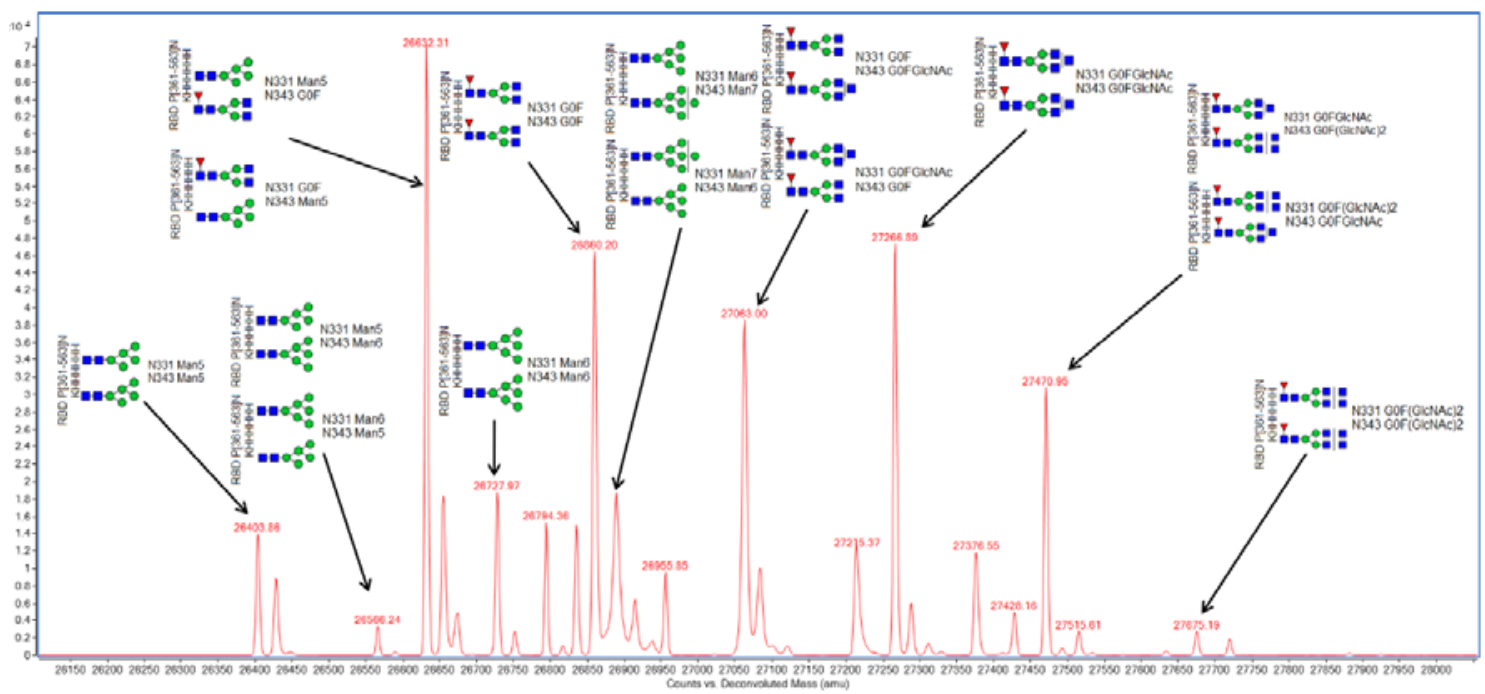

Elastase was chosen as a single digestion enzyme because it was judged to give the best chance of generating glycopeptides with a single NXS/T motif, essential for unambiguous glycan mapping. For non-glycosylated Spike peptides, elastase generated 63 high quality MSMS hits and 26\% coverage allowing for five missed cleavages. The same data searched for non-specific cleavage gave 135 high quality MSMS hits and $48 \%$ coverage allowing for twenty missed cleavages. Elastase itself contains 2 NXS/T motifs. We therefore prepared elastase only, at $x 10$ the usual concentration, searched the resulting LC-MS data using the PCDL as a control, and no hits were found. The Spike protein LC-MS data did contain a small number of elastase autodigestion peptides.

\section{Methods}

\section{Cloning, expression and purification of Spike}

The gene encoding amino acids 1-1208 of the SARS-CoV-2 Spike glycoprotein ectodomain (S), with mutations of RRAR > GSAS at residues 682-685 (to remove the furin cleavage site) and KV > PP at residues 986-987 (to stabilise the protein), was synthesised with a C-terminal T4 fibritin trimerization domain, HRV $3 \mathrm{C}$ cleavage site, $8 \times \mathrm{xHis}$ tag, and Twin-Strep-tag [5]. The construct was sub-cloned into pHL-sec [10] using the Agel and Xhol restriction sites and the sequence was confirmed by sequencing. Recombinant Spike was produced in Expi293F ${ }^{T M}$ cells by transient transfection with purified DNA (0.5 mg/L cells) using a 1:6 DNA:L-PEI ratio, mixed in minimal medium, and sodium butyrate as an additive. Cells were grown in suspension in FreeStyle $293^{T M}$ medium with shaking at $150 \mathrm{rpm}$ in $2 \mathrm{~L}$ smooth roller bottles, filled with $0.5 \mathrm{~L}$ cells at $2 \mathrm{e}^{6} / \mathrm{mL}$ per bottle at $30^{\circ} \mathrm{C}$ with $8 \% \mathrm{CO}_{2}$ and $75 \%$ humidity. Supernatants from transfected cells were harvested 3-days post-transfection by centrifugation. Clarified supernatant was mixed with $\mathrm{Ni}^{2+}$ IMAC Sepharose ${ }^{\circledast} 6$ Fast Flow (GE; $2 \mathrm{~mL}$ bed volume per $L$ of supernatant) at room temperature for $2 \mathrm{~h}$. Using a gravity flow column, resin 
was collected and washed stringently with $50 \mathrm{CV}$ each of base buffer (1X PBS), WB25 (BB + $25 \mathrm{mM}$ imidazole), and WB40 (BB+ $40 \mathrm{mM}$ imidazole), followed by elution with $\mathrm{EB}$ (0.30 M imidazole in $1 \mathrm{X}$ PBS). Protein was dialyzed into $1 X$ PBS using SnakeSkin ${ }^{T M} 3,500$ MWCO dialysis tubing, concentrated to $1 \mathrm{mg} / \mathrm{mL}$ using a 100,000 MWCO VivaSpin centrifugal concentrator (GE), and centrifuged at $21,000 \times g$ for 30 min to remove aggregates. The trimeric Spike protein was flash frozen in $\mathrm{LN}_{2}$ and stored at $-80^{\circ} \mathrm{C}$ until use. Final purified yield was $1 \mathrm{mg}$ of Spike protein per $\mathrm{L}$ of transfected cells.

Cloning, expression and purification of Receptor Binding Domain

The receptor binding domain (RBD; aa 330-532) of SARS-CoV-2 Spike (Genbank MN908947) was inserted into the pOPINTTGneo expression vector fused to an $\mathrm{N}$-terminal signal peptide and a $\mathrm{C}$ terminal $6 \times \mathrm{His}$ tag [11]. RBD was produced by transient transfection in Expi293F ${ }^{T M}$ cells (ThermoFisher Scientific, UK) using purified DNA (1.0 mg/L cells), a 1:3 DNA:L-PEI ratio, and sodium butyrate as an additive. Cells were grown in suspension in FreeStyle $293^{\text {TM }}$ expression medium at $37^{\circ} \mathrm{C}$ with $8 \% \mathrm{CO}_{2}$ and $75 \%$ humidity. Supernatants from transfected cells were harvested 3-days posttransfection and the supernatant was collected by centrifugation. Clarified supernatant was incubated with $5 \mathrm{~mL}$ of $\mathrm{Ni}^{2+}$ IMAC Sepharose ${ }^{\circledR} 6$ Fast Flow $(G E)$ at room temperature for $2 \mathrm{~h}$. Using gravity flow, resin was washed with $50 \mathrm{CV}$ of base buffer (1X PBS) and $50 \mathrm{CV}$ of WB (1X PBS + $25 \mathrm{mM}$ imidazole) before elution with EB (0.5 M imidazole in 1X PBS). Protein was concentrated using a 10,000 MWCO Amicon Ultra-15 before application to a Superdex $7516 / 600$ column preequilibrated with $1 \mathrm{X}$ PBS pH 7.4. Peak monomeric fractions were pooled and concentrated to 2 $\mathrm{mg} / \mathrm{mL}$, flash frozen in $\mathrm{LN}_{2}$, and stored at $-80^{\circ} \mathrm{C}$ until use. Final purified yield was $>15 \mathrm{mg} R B D$ per $\mathrm{L}$ of transfected cells.

\section{Sample preparation}

SARS-CoV-2 Spike or RBD-6H at $1 \mathrm{mg} / \mathrm{mL}$ in PBS were prepared in aliquots of either $20 \mu \mathrm{L}$ or $80 \mu \mathrm{L}$ and diluted 1 in 3 in $100 \mathrm{mM}$ ammonium bicarbonate, $\mathrm{pH} 8.0$, followed by reduction by addition of 1, 4 Dithiothreitol (DTT) to $5 \mathrm{mM}$ and incubation $37^{\circ} \mathrm{C}$ for $1 \mathrm{~h}$. Next, the protein was alkylated by addition of iodoacetamide (IAA) to $15 \mathrm{mM}$ and incubation in the dark for $30 \mathrm{~min}$. This was followed by overnight digestion using elastase (Promega) at a ratio of 1:20 (w/w). The following day, the supernatant was dried using a rotary evaporator, and re-suspended in $60 \mu \mathrm{L}$ of $0.1 \%$ formic acid for injection into the LC-MS.

\section{'Analytical mode' LC-MS glycopeptide data acquisition}

LC-MS 'analytical mode' was performed using a 1290 Infinity UHPLC coupled to a G6530A ESI QTOF mass spectrometer (Agilent Technologies). TOF and quadrupole were calibrated prior to analysis and the reference ion $922.0098 \mathrm{~m} / z$ was used for continuous mass correction. Sample was introduced using a $50 \mu \mathrm{L}$ full-loop injection. Reversed phase chromatographic separation was achieved using an AdvancedBio Peptide reversed phase $2.7 \mu \mathrm{m}$ particle, $2.1 \mathrm{~mm} \times 100 \mathrm{~mm}$ column 655750-902 (Agilent Technologies). Mobile phase A was $0.1 \%$ formic acid in water and mobile phase B $0.1 \%$ formic acid in methanol (Optima LC-MS grade, Fisher). Initial conditions were $5 \%$ B and $0.200 \mathrm{~mL} / \mathrm{min}$ flow rate. A linear gradient from 5\% B - 60\% B was applied over $60 \mathrm{~min}$, followed by isocratic elution at $100 \%$ B for 2 min returning to initial conditions for a further $2 \mathrm{~min}$. Post time was $10 \mathrm{~min}$. MS source parameters were drying gas temperature $350^{\circ} \mathrm{C}$, drying gas $8 \mathrm{~L} / \mathrm{min}$, nebulizer 30 psi, capillary 4000 $\mathrm{V}$, fragmentor $150 \mathrm{~V}$. MS spectrum range was $100-3200 \mathrm{~m} / z$ (centroid only), $2 \mathrm{GHz}$ Extended Dynamic range, with the instrument in positive ion mode.

\section{LC-MSMS glycopeptide data acquisition 'discovery mode'}

LC-MSMS 'discovery mode' was performed as described above, with the following changes: Soft CID collision energy parameters for MSMS were slope 1.0, intercept 0 using argon as the collision gas (if using nitrogen slope 2.0, intercept 0 ) were used to favour glycan fragmentation over peptide 
fragmentation for glycopeptides. Sufficient non-glycosylated peptides were fragmented to give reasonable sequence coverage. Care was taken to reduce sodium and potassium contamination where possible and Tris buffers were avoided as these adducts interfere with glycopeptide analysis.

\section{LC-MS glycopeptide data analysis 'analytical mode'}

Analysis only required retention time and accurate mass data using the Spike PCDL database created as described below. This is possible either using the Agilent software described, software provided by other vendors, or by manual inspection. In our case, we used Masshunter Qualitative Analysis version B.07 (Agilent Technologies) and the Molecular Feature Extraction tool to extract $\mathrm{H}+, \mathrm{Na}+$ and $\mathrm{K}+$ adducts and charge states +1 to +5 . Briefly, this tool identifies and associates common spectral features such as carbon isotopes, adducts and multiple charge states as belonging to same Compound (peptide) by virtue of sharing the same accurate mass and retention time, then combines these features together to give a mass, retention time and volume for each compound. Compounds were then searched against Spike PCDL using a mass error window $+/-10 \mathrm{ppm}$ and a retention time window $+/-2$ min. Some filtering of the data was used to reduce the number of compounds and thence speed-up the PCDL search. Relative quantitation of each glycan on a particular glycopeptide could then be assessed.

\section{LC-MSMS glycopeptide analysis "discovery mode"}

Construction of the Spike glycopeptide mass-retention time database ("discovery mode") was more complex and time-consuming, but once constructed and made available to the scientific community, there is no further need to repeat this step. By using reverse phase HPLC, glycopeptides are separated by the relatively hydrophobic peptide moiety, whereas the associated hydrophilic glycans are grouped together by retention time as illustrated in figure 7 .

Figure 7. LC-MSMS "discovery" mode used to generate the Spike glycopeptide Mass-Retention Time PCDL database

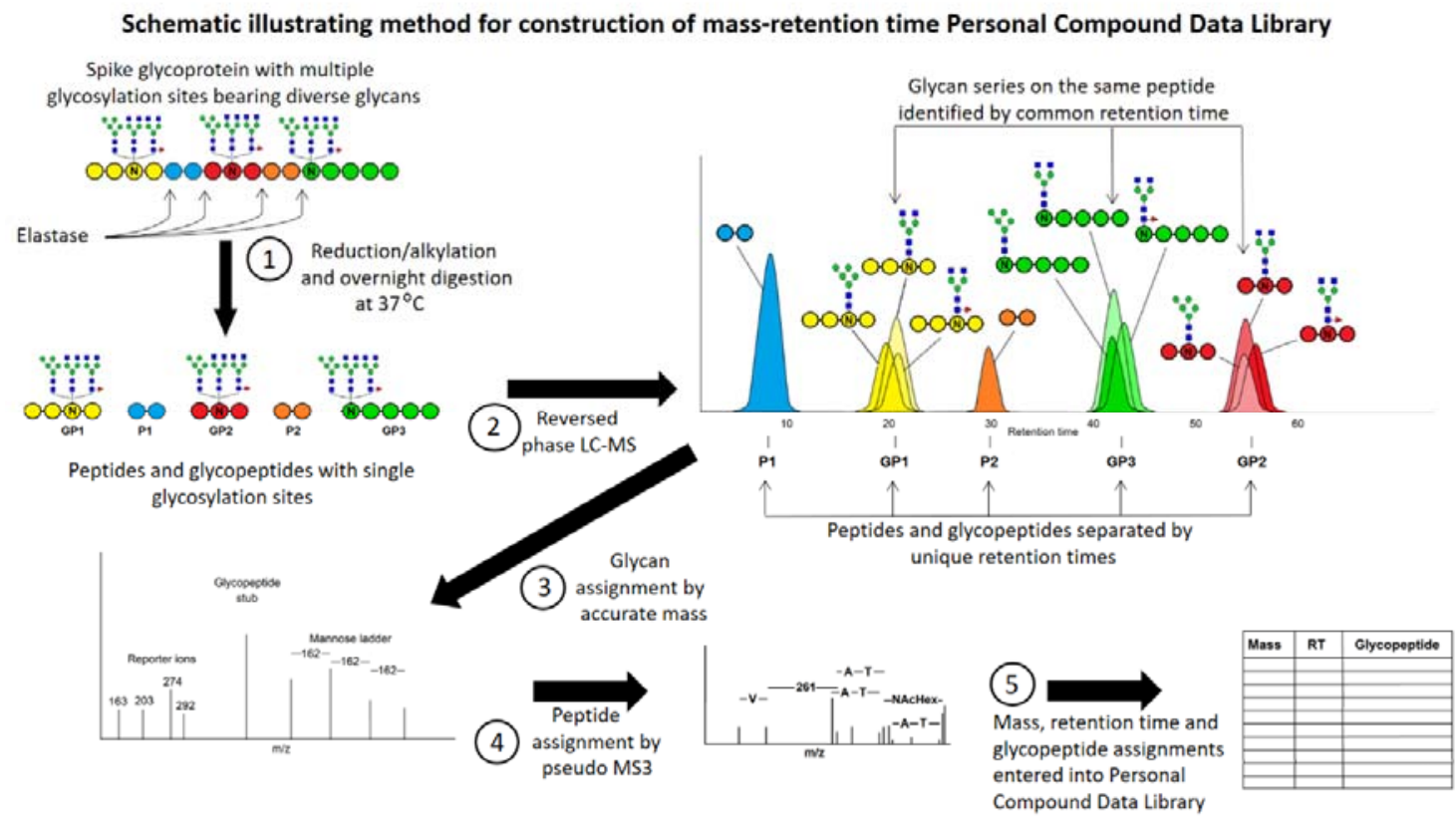

Initial LC-MSMS discovery mode data for incorporation into a glycopeptide PCDL was performed using Masshunter Qualitative Analysis with Bioconfirm B.07.00 (Agilent Technologies). Compounds were identified using the Find by Molecular Feature (MFE) tool looking for $\mathrm{H}+, \mathrm{Na}+$ and $\mathrm{K}+$ adducts 
and charge states +2 to +5 . The results were filtered to remove compounds $<1000 \mathrm{Da}$ (too small to be glycopeptides). Compound MSMS spectra were screened manually for the following oxonium reporter ions: Hex $m / z$ 163.0601, HexNAc $m / z$ 204.0866, HexHexNAc $m / z$ 366.1395, Neu5Ac $m / z$ $274.0921 / \mathrm{m} / z 291.0949$ and/or a Hexose ladder ${ }_{\delta} \mathrm{M} 162.0528 \mathrm{Da}$. High quality $\mathrm{m} / \mathrm{z}$ spectra were deconvoluted to neutral mass spectra with glycan de novo interpretation performed manually. Once a glycopeptide had been identified, it was entered into a personal compound data library database (PCDL, Agilent Technologies) as a mass and retention time. In addition, the database made use of known mammalian $\mathrm{N}$-linked glycan processing. After the initial glycopeptide identification, other processed glycopeptides, which were considered likely to also be present, were added to the database at the same retention time and with a calculated mass. For example, if a glycopeptide with Man5 was identified by MSMS, Man1-9 and G0/F were added at the same retention time. If these glycans were subsequently found in the data, their actual retention times were updated, and the next round of processing to more complex glycans was added, in order to produce the most comprehensive PCDL possible, while still being manageable. Processing order:

$$
\operatorname{Man}(\mathrm{n}) \rightarrow \mathrm{G} 0 / \mathrm{F} \rightarrow \mathrm{G} 1 / \mathrm{F} \rightarrow \mathrm{G} 2 / \mathrm{F} \rightarrow \mathrm{A} 1 / \mathrm{F} \rightarrow \mathrm{A} 2 / \mathrm{F} \rightarrow \text { Very Complex }
$$

Valid glycan identifications resulted in a calculated peptide mass that could be matched to the sequence. Where high quality spectra were present, a peptide-GIcNAc stump was observed (Figure 4). This was used in a pseudo MS3 experiment with manual peptide de novo interpretation to confirm the peptide sequence (Figure 5). Mass data adjacent to the glycopeptide retention time was then searched for neutral differences corresponding to glycans, for example, Man5 $\rightarrow$ GOF or Man7 $\rightarrow$ G2F has a neutral delta mass of 228.1111 Da.

As expected, not all species could be matched to the sequence, presumably due to unexpected modifications. In this case, they were added to the database as ' $G P$ ' with an identifying number and as much information as could be extracted. Data for the most likely glycan was added to the PCDL, including a deconvoluted mass MSMS spectra were available, using nomenclature generating the most easily readable format.

A second round of glycopeptide discovery used Bioconfirm v10.0 data analysis software (Agilent Technologies). Sequences were matched by peptide accurate mass using the following parameters: peptide cleavage nonspecific, number of missed cleavages 20, N-linked modifications Man3, Man59, G0, GOF, GOF GICNAc, G1, G1F, G2, G2F. Any peptide bearing the glycosylation motif NXS/T with two or more glycan hits within a retention time window $+/-2$ min was added to the PCDL, excepting missed cysteine alkylations.

In-source fragmentation due to glycopeptide ions absorbing excess energy could be identified in the MS by searching extracted ion chromatograms (EICS) of the oxonium reporter ions and also by related glycopeptides appearing with exactly the same retention times. Both were observed infrequently and at manageable levels.

Intact mass analysis

Concentrated protein samples were diluted to $0.02 \mathrm{mg} / \mathrm{mL}$ in $0.1 \%$ formic acid and $50 \mu \mathrm{L}$ was injected on to a $2.1 \mathrm{~mm} \times 12.5 \mathrm{~mm}$ Zorbax $5 \mu \mathrm{m} 300 \mathrm{SB}-\mathrm{C} 3$ guard column (Agilent Technologies) housed in a column oven set at $40^{\circ} \mathrm{C}$. The solvent system used consisted of $0.1 \%$ formic acid (solvent A) and $0.1 \%$ formic acid in methanol (solvent $B$ ). Chromatography was performed as follows: Initial conditions were $90 \% A$ and $10 \% B$ and a flow rate of $1.0 \mathrm{~mL} / \mathrm{min}$. A linear gradient from $10 \% \mathrm{~B}$ to $80 \%$ B was applied over 35 seconds. Elution then proceeded isocratically at $95 \%$ B for 40 seconds followed by equilibration at initial conditions for a further 15 seconds. The mass spectrometer was configured with the standard ESI source and operated in positive ion mode. The ion source was operated with the capillary voltage at $4000 \mathrm{~V}$, nebulizer pressure at $60 \mathrm{psig}$, drying gas at $350^{\circ} \mathrm{C}$ and 
drying gas flow rate at $12 \mathrm{~L} / \mathrm{min}$. The instrument ion optic voltages were as follows: fragmentor 250 $\mathrm{V}$, skimmer $60 \mathrm{~V}$ and octopole RF $250 \mathrm{~V}$.

\section{Discussion}

Glycoprotein analysis is difficult. It is either performed in biopharmaceutical laboratories with proprietary expertise of glycan analysis on simple glycoproteins, such as immunoglobulins, or performed by a handful of academic labs with experience of glycan discovery from complex glycoproteins. Many protein researchers choose to ignore it, manipulating cell lines such that they cannot process beyond Man5, or to remove glycans entirely by mutation at the glycosylation motif or enzymatically [12]. While this approach has its merits, it has exposed a serious weakness in analytical capability when faced with a pathogen such as SARS-CoV-2 whose ability to evade the immune system is dependent upon heavy and complex glycosylation.

We have chosen an approach relying on elastase digestion to generate glycopeptides bearing a single glycan but with a sufficient number of amino acid residues to enable chromatographic separation by reversed-phase HPLC, as well as confident identification by accurate mass or de novo sequencing. Our choice of reversed phase HPLC has excellent discrimination for short elastase peptides, whereas glycans show little or no interaction with the column. Thus, species originating from a single glycosylation site with the same peptide sequence but several different glycans, eluted with the same retention time and could be discriminated by mass spectrometry. We used reversed phase HPLC and MSMS to characterise as many glycopeptides as possible. Although this required complex and time-consuming data analysis, it needed only be performed once, with the goal of building an accurate mass-retention time database for all observed Spike glycopeptides. Provided the same HPLC column and mobile phase conditions are used, retention times should not vary significantly. Thus, working in the analytical mode we describe, glycan structure and peptide sequence is assigned confidently, by accurate mass and retention time alone. LC-MS data need only to be searched against the mass-retention time database, and peak areas recorded, to generate a complete characterisation of Spike glycans.

We believe the MRTF method described here has advantages over other approaches to Spike glycan analysis. Previous studies relied upon very expensive equipment and software unavailable in most analytical laboratories. Working in 'analytical' mode, all that is necessary is to reproduce the chromatography, hence our method is a generic one, which can be run using any HPLC coupled to any accurate mass instrument and is not restricted to specific proprietary data analysis software. We used PCDL and Masshunter, but MRTF analysis can be performed on any vendor software or manually. Moreover, it demands no specialised expertise in glycobiology, and is thus accessible to many more researchers. Some published methods require multiple specific endoproteases, some of which cannot be readily sourced. Our method uses a single enzyme, elastase, which is inexpensive and widely available. Nor does it rely on glycosidases, which may not work efficiently and do not cleave O-linked glycans.

Our data contains an excess of glycopeptides with the motif $(\mathrm{y}) \mathrm{nNxS} / \mathrm{T}$. This appears to be a very convenient function of elastase on glycopeptides, because the presence of the motif at the Cterminus facilitates de novo sequencing. We would be interested to know if this cleavage bias towards the C-terminus of the glycan motif is reproducible in other labs and whether it indicates steric hindrance within the elastase enzyme structure. If such bias is real, then these peptides are less likely to be a false positive result.

Receptor binding domain (RBD) from Spike protein is of interest in many labs for development of serological tests or neutralising antibodies. Because the yield of RBD was five times higher than Spike and more was initially available, we used it for method optimisation, and since it bears only two glycosylation sites which are also present on Spike, it functioned as a useful model. Consequently, N343 on glycopeptide GEVFNAT is over-represented in our demonstration PCDL. We consistently 
observed the same three major glycans (Man5, GOF and GOF+GluNAc) on this peptide and these were also in agreement with intact mass analysis of RBD protein as shown in Figure 6. On closer inspection, glycans up to A2F could also be observed at lower levels. We suspect that sufficiently detailed analysis may reveal all possible glycan structures with low abundance at all available sites. The most important would therefore be the top three to five glycans. If the complete complement of Spike protein glycoforms proves too challenging for a single analysis, this site, which is the most complete, would make a good proxy for total glycosylation.

We acknowledge that the mass-retention time fingerprinting method described, like all database searching methods, is dependent on the reproducibility of the enzyme digestion and both the quality and the completeness of database being searched. The example PCDL database reported here is provided as a demonstration. Due to glycan complexity and the likely absence of specific glycans within the Spike batches prepared by us, it will always be incomplete. Moreover, individual glycopeptides were identified with variable degrees of certainty, and we recommend that they should be validated by the user. As with all glycan analysis methods, there is a bias towards glycopeptides that are easiest to identify by the techniques used, and such bias will also be reflected within the database. Once the PDCL has been created, it must be refined and extended over time to improve data quality, and it is our intention to do so.

\section{References}

1. Zhang, Y., et al., Site-specific $\mathrm{N}$-glycosylation Characterization of Recombinant SARS-CoV-2 Spike Proteins using High-Resolution Mass Spectrometry. bioRxiv, 2020.

2. Solá, R.J. and K. Griebenow, Glycosylation of therapeutic proteins. BioDrugs, 2010. 24(1): p. 9-21.

3. Vugmeyster, Y., et al., Pharmacokinetics and toxicology of therapeutic proteins: advances and challenges. World journal of biological chemistry, 2012. 3(4): p. 73.

4. Tortorici, M.A. and D. Veesler, Structural insights into coronavirus entry. Advances in virus research, 2019. 105: p. 93-116.

5. Wrapp, D., et al., Cryo-EM structure of the 2019-nCoV spike in the prefusion conformation. Science, 2020. 367(6483): p. 1260-1263.

6. Yang, T.-J., et al., Cryo-EM analysis of a feline coronavirus spike protein reveals a unique structure and camouflaging glycans. Proceedings of the National Academy of Sciences, 2020.

7. Shajahan, A., et al., Deducing the $\mathrm{N}$-and O-glycosylation profile of the spike protein of novel coronavirus SARS-CoV-2. bioRxiv, 2020.

8. Watanabe, Y., et al., Site-specific analysis of the SARS-CoV-2 glycan shield. BioRxiv, 2020.

9. Yao, H., et al., Molecular architecture of the SARS-CoV-2 virus. bioRxiv, 2020: $\mathrm{p}$. 2020.07.08.192104.

10. Aricescu, A.R., W. Lu, and E.Y. Jones, A time-and cost-efficient system for high-level protein production in mammalian cells. Acta Crystallographica Section D: Biological Crystallography, 2006. 62(10): p. 1243-1250.

11. Nettleship, J.E., et al., Transient expression in HEK 293 cells: an alternative to E. coli for the production of secreted and intracellular mammalian proteins, in Insoluble proteins. 2015, Springer. p. 209-222.

12. Chang, V.T., et al., Glycoprotein structural genomics: solving the glycosylation problem. Structure, 2007. 15(3): p. 267-273.

\section{Acknowledgements}

The authors wish to thank Professor David Harvey for critical reading of the manuscript and for helpful comments. We thank Professor Ray Owens for kindly providing the RBD-6H construct and Professor Gavin Screaton and Dr. Juthathip Mongkolsapaya for kindly providing the Spike construct. AdvancedBio Peptide HPLC column was a gift from Agilent Technologies. This project has received 
bioRxiv preprint doi: https://doi.org/10.1101/2020.07.24.217562; this version posted July 27, 2020. The copyright holder for this preprint (which was not certified by peer review) is the author/funder, who has granted bioRxiv a license to display the preprint in perpetuity. It is made available under aCC-BY-ND 4.0 International license.

funding from the Innovative Medicines Initiative 2 Joint Undertaking (JU) under grant agreement No 875510. The JU receives support from the European Union's Horizon 2020 research and innovation programme and EFPIA and Ontario Institute for Cancer Research, Royal Institution for the Advancement of Learning McGill University, Kungliga Tekniska Hoegskolan, Diamond Light Source Limited. The SGC is a registered charity (number 1097737) that receives funds from AbbVie, Bayer Pharma AG, Boehringer Ingelheim, Canada Foundation for Innovation, Eshelman Institute for Innovation, Genentech, Janssen, Merck KGaA, Darmstadt, Germany, MSD, Ontario Ministry of Research, Innovation and Science (MRIS), Pfizer, São Paulo Research Foundation-FAPESP, Takeda, and Wellcome [106169/ZZ14/Z]. 